\title{
Polarization Aberration in Astronomical Telescopes
}

\author{
Russell A. Chipman*a, Wai Sze Tiffany Lam ${ }^{\mathrm{a}}$, James Breckinridge ${ }^{\mathrm{a}, \mathrm{b}}$ \\ ${ }^{a}$ University of Arizona, College of Optical Sciences, 1630 E. University Drive, Tucson, AZ, USA \\ 85718; ${ }^{b}$ Graduate Aerospace Laboratories, California Institute of Technology, 1200 E. California \\ Blvd., Pasadena, CA, USA 91125.
}

\begin{abstract}
The point spread function (PSF) for astronomical telescopes and instruments depends not only on geometric aberrations and scalar wave diffraction, but also on the apodization and wavefront errors introduced by coatings on reflecting and transmitting surfaces within the optical system. The functional form of these aberrations, called polarization aberrations, result from the angles of incidence and the variations of the coatings as a function of angle. These coatings induce small modifications to the PSF, which consists of four separate components, two nearly Airy-disk PSF components, and two faint components, we call ghost PSF components, with a spatial extent about twice the size of the diffraction limited image. As the specifications of optical systems constantly improve, these small effects become increasingly important. It is shown how the magnitude of these ghost PSF components, at $\sim 10^{-5}$ in the example telescope, can interfere with exoplanet detection with coronagraphs.
\end{abstract}

Keywords: Polarization, imaging, polarization aberration, polarization ray tracing

\section{INTRODUCTION}

The aberration of an optical system is its deviation from ideal performance. In an imaging system with ideal spherical or plane wave illumination, the desired output is spherical wavefronts with constant amplitude and constant polarization state centered on the correct image point. The deviations from spherical wavefronts are known as the wavefront aberration function. Deviations from constant amplitude arise from differences in reflection or refraction efficiency between rays. Amplitude variations cause amplitude aberration or apodization. At each reflecting and refracting surface, polarization change also occurs due to differences between the $p$ and $s$-reflectance and transmission coefficients. Over each wavefront, the angles of incidence changes causing polarization variation, so that a uniformly polarized beam acquires polarization variations exiting the system..$^{[1],[2]}$ The ideal output polarization for most systems would be a non-varying polarization state; there would be no polarization change propagating from object to image space. Such ray paths through a (non-polarizing) optical system can be characterized with Jones matrices which would be identity matrices for all ray paths. Deviations from this identity matrix function are called polarization aberrations.

In high performance astronomical systems, the variations of polarization and amplitude have a much smaller impact on the image quality than the wavefront aberrations. However as the astronomical community prepares to image and measure the spectrum and polarization of exoplanets, and the microlithography community closes in on $10 \mathrm{~nm}$ spatial resolution, such small polarization and amplitude effects as these must be considered. To calculate polarization matrices for ray paths through optical systems, the technique of polarization ray tracing was developed. ${ }^{[2]-[8]}$ Vector extensions to diffraction theory then allow the diffraction patterns and image quality of polarization-aberrated beams to be simulated. ${ }^{[1],[9]-[15]}$ The functional form of these polarization aberrations frequently have similar patterns to the geometrical aberrations, since they arise from the geometry of surfaces and the variation of angle of incidence. ${ }^{[11],[12],[16]-[23]}$

\section{IMAGE FORMATION}

Figure 1 describes the steps in the calculation of image formation with polarization aberration. In the following, matrix functions are described with bold acronyms. The Jones pupil is determined as an array of Jones matrix values by polarization ray tracing. The Jones pupil plays the role of the wavefront aberration function of geometrical optics; it is comprised of four wavefront aberration functions and four amplitude functions for four polarization combinations. The amplitude response function of geometrical optics is replaced with an Amplitude Response Matrix (ARM), calculated as the Fourier transforms of the Jones pupil elements. The Point Spread Matrix (PSM) for an image of an incoherent point source is calculated by transforming the Jones matrices of the ARM into Mueller matrices. ${ }^{[24]-[26]}$ The point image flux and

*rchipman@optics.arizona.edu; phone 1520626 9435; http://fp.optics.arizona.edu/chipman/

Polarization Science and Remote Sensing VII, edited by Joseph A. Shaw,

Daniel A. LeMaster, Proc. of SPIE Vol. 9613, 96130H · () 2015 SPIE

CCC code: $0277-786 \mathrm{X} / 15 / \$ 18 \cdot$ doi: $10.1117 / 12.2188921$

Proc. of SPIE Vol. $961396130 \mathrm{H}-1$ 
polarization distribution is a function of the four Stokes parameters obtained by matrix multiplying the Stokes parameters by the PSM. ${ }^{[1]}$

\begin{tabular}{|c|c|c|c|}
\hline Fourier Transform & ARM & $\begin{array}{c}\text { Jones } \\
\text { Matrix }\end{array} \rightarrow \begin{array}{l}\text { Mueller } \\
\text { Matrix }\end{array}$ & PSM \\
\hline 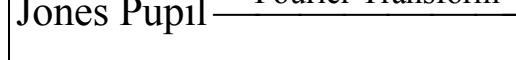 & Amplitude Response Matrix & & ${ }^{\rightarrow}$ Point Spread Matrix \\
\hline
\end{tabular}

Figure 1. The steps in evaluating the image quality of polarization aberrated optical systems. The Fourier transform of the Jones pupil is the ARM. The Jones matrix ARM converts into a Mueller matrix PSM to evaluate incoherent image quality, such as the image of a star in an astronomical telescope.

\section{EXAMPLE CASSEGRAIN TELESCOPE}

In this manuscript, the polarization image formation of a generic telescope is analyzed. It is difficult to choose a fully representative astronomical optical system as an example. If an example system with too many elements is selected, it is more difficult to relate the individual surfaces to the features in the polarization aberration and polarized PSF, so a relatively simple system of a Cassegrain telescope and fold mirror illuminated on-axis, as shown in Figure 2, is analyzed. The specific values for the telescope are interesting to gauge the order of magnitude of the polarization effects in similar telescopes. But the even more interesting results are the functional form of the image defects. This telescope was chosen to have no on-axis geometric wavefront aberrations; the optical path lengths $(O P L s)$ for all on-axis rays are equal. The image calculated by conventional geometrical ray tracing is ideal, so deviations from the ideal image result from the polarization of the mirrors since the effects of wavefront aberration is zero. The polarization ray trace assumes mirrors coated with aluminum with a refractive index $N=2.80+8.45 i$ and analyzed at $800 \mathrm{~nm}$. Figure 3 plots the Fresnel amplitude and phase coefficients for aluminum which are used throughout the remainder of this manuscript which determines their effect on the example telescope's image quality, and provides an example for other image forming systems.

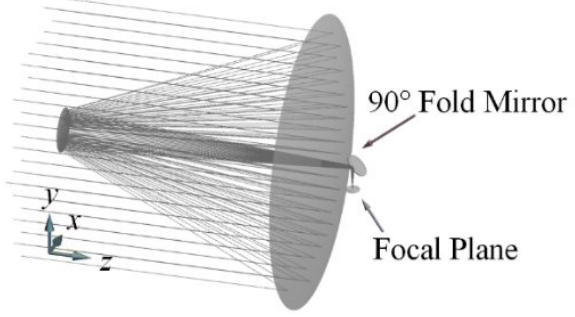

Figure 2. An example Cassegrain telescope system with a primary mirror at F/1.2, a Cassegrain focus of $\mathrm{F} / 8$, and a $90^{\circ}$ fold mirror in the $\mathrm{F} / 8$ converging beam. The $90^{\circ}$ fold mirror is folded about the $x$-axis. The primary mirror has a clear aperture of 2.4 meters. The operating wavelength is $800 \mathrm{~nm}$. All three mirrors are coated with aluminum. $x$ and $y$ define the coordinates for incident polarization states.

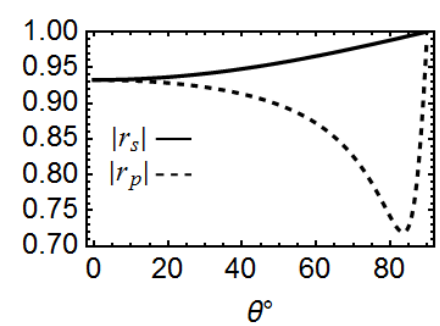

(a)

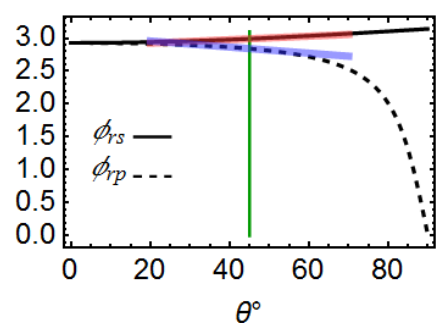

(b)

Figure 3. Reflection coefficients for the amplitude (a) and the phase (b) upon reflection at angle of incidence $\theta^{\circ}$ between $0^{\circ}$ and $90^{\circ}$ for a bare aluminum mirror at $800 \mathrm{~nm}$ wavelength. In (b), $\phi_{r s}$ and $\phi_{r p}$ are the reflected phase for $s$ and $p$-polarized light. The green vertical line highlights the reflection phase at $45^{\circ}$. The red and blue lines show the corresponding slope of $\phi_{r s}$ and $\phi_{r p}$ at the $45^{\circ}$ incident angle. 
Metal mirrors act as weak polarizers, or diattenuators, due to the difference between $\left|r_{s}\right|^{2}$ and $\left|r_{p}\right|^{2}$. This is characterized by the diattenuation $D$,

$$
D=\frac{\left|r_{s}\right|^{2}-\left|r_{p}\right|^{2}}{\left|r_{s}\right|^{2}+\left|r_{p}\right|^{2}} .
$$

Metal mirrors also act as retarders due to the phase shift $\delta$ between the $s$ and $p$-reflected beams,

$$
\delta=\left|\phi_{r p}-\phi_{r S}\right| \text {. }
$$

The maximum fraction $F$ of light which can be coupled into the orthogonal polarization state for small diattenuation (dimensionless) or small retardance (radians), occurs for light at $45^{\circ}$ or $135^{\circ}$ to the retardance fast axis or the diattenuation axis. The coupling for both effects share the same quadratic form for $F$,

$$
F(D) \approx \frac{D^{2}}{4}, F(\delta) \approx \frac{\delta^{2}}{4} .
$$

Using the Mueller calculus, these equations are readily derived: place a diattenuator or retarder oriented at $45^{\circ}$ between crossed polarizers, and evaluate the transmitted flux as a Taylor series.

The example telescope's polarization has been calculated with the polarization ray tracing program Polaris-M developed by R. Chipman and colleagues at the University of Arizona. Maps of diattenuation for each of the three mirrors are shown in the three left panels of Figure 4. The Figure 4 right panel maps the cumulative diattenuation viewed looking into the exit pupil. Each line indicates the diattenuation magnitude and the orientation of the state with maximum transmission at a grid of locations in the pupil. In the first two panels in Figure 4, it is seen that the primary and secondary mirrors have diattenuation which is rotationally symmetric, tangentially oriented. The magnitude of the diattenuation increases quadratically from the center to the edge of the pupil. Throughout the manuscript the terms linear and quadratic, etc. mean approximately linear and approximately quadratic; this is standard use in aberration theory. The fold mirror has a very different functional form. It introduces a horizontally oriented diattenuation with a linear variation along a vertical axis. On the right, the cumulative diattenuation map is similar to the fold mirror, predominantly linear from top to bottom, because the fold mirror has the largest diattenuation magnitude.
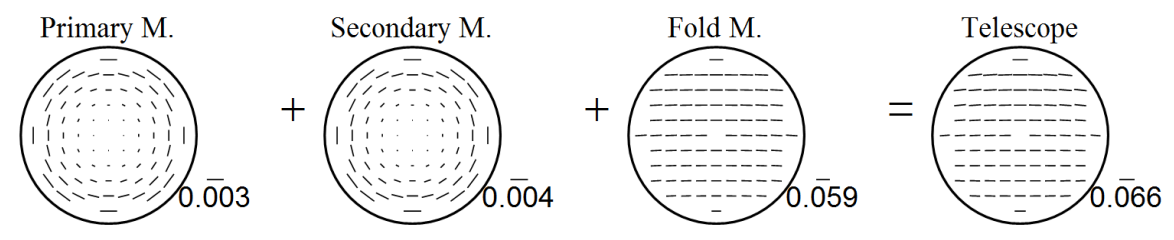

Figure 4. Diattenuation maps where length of each line is proportional to the magnitude of the diattenuation. The orientation of the lines shows the axis of maximum transmission. Each individual mirror is shown in the first three panels. The cumulative diattenuation is in the last panel. The scale in the lower right corner of each panel shows the magnitude of the largest diattenuation. For this example telescope, the dominant source of diattenuation is the $90^{\circ}$ fold mirror.
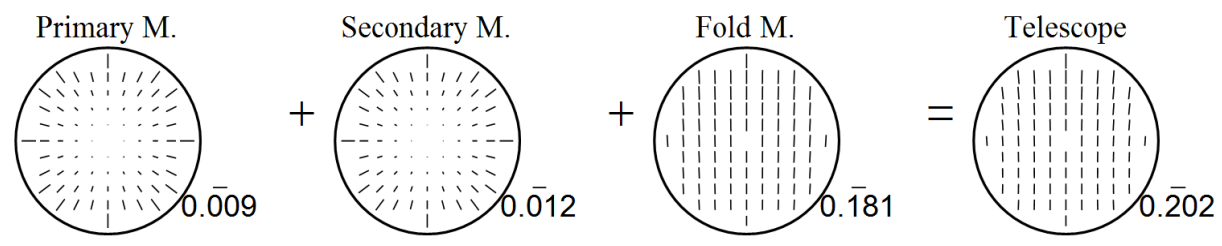

Figure 5. The first three panels show retardance maps for individual mirror elements. The last panel is the cumulative retardance. Length indicates retardance magnitude and orientation indicates the fast axis. The scale of the largest retardance in radians is in the lower right corner. The dominant source of retardance for the telescope is the $90^{\circ}$ fold mirror (second panel from the right). 
Figure 5 shows the retardance aberration maps. It is important to understand that metal's retardance introduces a polarization dependent phase contribution into the $O P L$ which is different for $s$ and $p$-polarizations. This yields a difference in the metal coatings' contributions to the wavefront aberration contributions. The fold mirror has a linearly varying retardance increasing from the bottom to the top of the pupil. The resultant retardance for the entire telescope (right) is dominated by the fold mirror's retardance; the primary and secondary mirrors make smaller contributions. The linear variation of retardance causes a difference in the wavefront aberration tilt, so the $X$ and $Y$-polarizations have different linear phases, and the $X$ and $Y$-images are shifted by different amounts from the nominal image location.

The polarization change of each ray through the optical system has an associated Jones matrix from which the diattenuation and retardance were calculated. The set of Jones matrices expressed as a function of pupil coordinates and object coordinates is called the polarization aberration function. ${ }^{[1]}$ The set of Jones matrices at each point in the pupil $(x, y)$ for a specified object point is named Jones pupil, and takes the form of a $2 \times 2$ Jones matrix pupil map with complex distributions of amplitude $A(x, y)$ and phase $\phi(x, y),{ }^{[27]}$,

$$
\mathbf{J}=\left(\begin{array}{cc}
J_{X X} & J_{X Y} \\
J_{Y X} & J_{Y Y}
\end{array}\right)=\left(\begin{array}{cc}
A_{X X} e^{i \phi_{X X}} & A_{X Y} e^{i \phi_{X Y}} \\
A_{Y X} e^{i \phi_{Y X}} & A_{Y Y} e^{i \phi_{Y Y}}
\end{array}\right) .
$$

For an $X$-polarized incident field at the entrance pupil, $A_{X X}$ is the amplitude of the $X$-polarized field at point $(x, y)$ in the exit pupil. Also, for the $X$-polarized incident field at the entrance pupil, $A_{Y X}$ is the amplitude at point $(x, y)$ of light coupled from $X$ into the $Y$-polarized field at the exit pupil. The term $\phi_{X X}$, the complex argument of $J_{X X}$, is the phase shift from the $X$ polarized incident field to the $X$-polarized exiting field due to the metal reflections. $\phi_{X X}$ is one of four wavefront aberration terms, in this case what would be measured by an interferometer illuminating with $X$-polarized light, and analyzing with an $X$-polarizer. The term $\phi_{Y X}$ is the phase shift for the $X$-polarized field coupled into the $Y$-polarized field. Similarly the right column of $\mathbf{J}$ describes the effects for the $Y$-polarized incident field. Putting all of this together, the field from a single point in object space maps into the exit pupil is described by the $2 \times 2$ Jones matrix in Eq. (4).

For the description of the Jones matrices, a coordinate system must be chosen for both the input light and the output light. The choice of the orthogonal basis is arbitrary, but it is simplest to decompose the incident plane waves into a component parallel to our fold mirror's rotation axis, horizontal or $X$-polarized, and a vertical or $Y$-polarized component. Then these basis states are followed through a non-polarizing optical system to define the $X$ and $Y$-components in the exit pupil. The result of all flux and PSF calculations, since they are intensities, are independent of the orthogonal basis chosen.

The result of the polarization ray trace is the Jones pupil shown in Figure 6. This is color coded to show the amplitude and phase variations across the exit pupil. Because the polarization effects from the mirrors are relatively small, both the diattenuation and retardance are much less than one, the Jones pupil is close to the identity matrix times a constant, $\sim 0.806$; the 0.806 results from the aluminum's reflection losses.

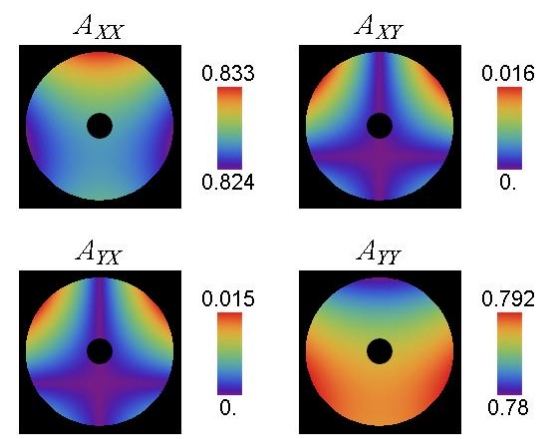

(a)

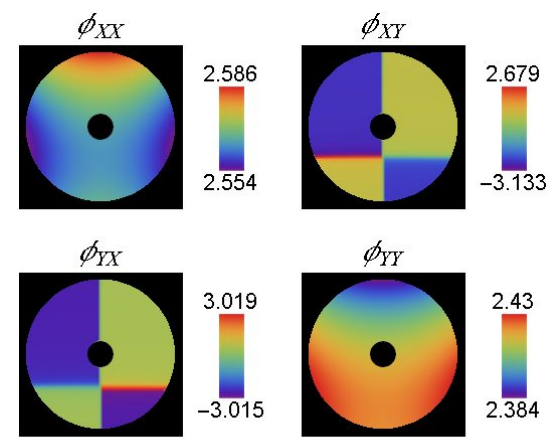

(b)

Figure 6. The Jones pupil elements displayed as color-coded images show the amplitude, phase, and polarization variation across the exit pupil. In (a), the four images show the amplitudes as functions of pupil position. The four images in (b) show the phase; these are wavefront aberration-like terms. $A_{X Y}$ and $A_{X Y}$ are strongly apodized so the corresponding diffraction patterns are much larger than near Airy-disk images associated with the diagonal terms. Each box has a scale to the right: units are amplitude for (a) and phase in radians for (b). A complex numbers phase changes by $\pi$ when the amplitude passes through zero. This is the cause of the $\phi_{X Y}$ and $\phi_{Y X}$ phase discontinuities. 
The phases of the Jones pupil's four elements represent wavefront aberration function contributions from the aluminum mirrors, as shown in Figure 6 (b). The Fresnel phase changes are different for the $s$ and $p$-components so they have different wavefronts. The telescope's on-axis geometrical wavefront aberration is zero. Therefore these phase variations across the pupil are wavefront contributions from the phase changes at the mirror coatings. $\phi_{X X}$, the wavefront aberration function of the telescope illuminated with $x$-polarized light and analyzed with an $x$-analyzer, has an overall linear variation of about 0.008 waves with a smaller additional deviation which is primarily astigmatism. The diagonal elements $\phi_{X X}$ and $\phi_{Y Y}$ each have a different wavefront tilt (linear component). This tilt difference arises at the fold mirror, seen in figure 3(b), where one can see that around the $45^{\circ}$ incident angle, and the phase slopes are different for $s$-incident polarized light vs. $p$-incident polarized light. If the Fresnel phases were linear about $45^{\circ}$, only tilt would be introduced. The small deviations from linear introduce higher order aberrations including small amounts of astigmatism (from quadratic deviation), coma (from cubic deviation) and other aberrations.

The image formed by optical systems with polarization aberration can be calculated with the ARM, a Jones calculus version of the amplitude response function from scalar diffraction theory,

$$
\mathbf{A R M}=\left(\begin{array}{ll}
\mathfrak{I}\left[J_{X X}(x, y)\right] & \mathfrak{I}\left[J_{X Y}(x, y)\right] \\
\mathfrak{I}\left[J_{Y X}(x, y)\right] & \mathfrak{I}\left[J_{Y Y}(x, y)\right]
\end{array}\right),
$$

where $\mathfrak{I}$ is a spatial Fourier transform applied to each of the Jones pupil elements. The ARM for the example telescope of Figure 2 is shown in Figure 7. Table 1 summarizes the system and lists several parameters relevant to the imaging calculations.

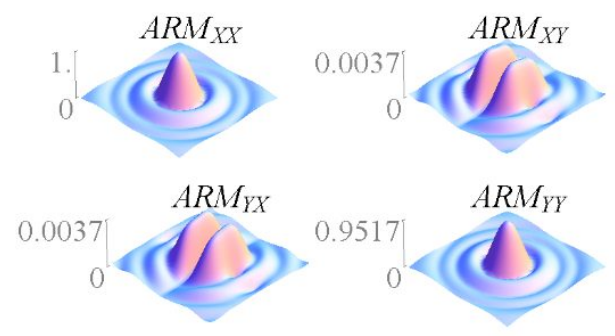

Figure 7. The absolute value of the amplitude of the $2 \times 2 \mathbf{A R M}$ at an on-axis field point is shown for the example telescope of Figure 2 normalized by the peak of the $X X$-component.

The ARM's diagonal elements are close to the Airy disk, but enlarged a little due to the aberrations in $\phi_{X X}$ and $\phi_{Y Y}$. Both patterns are slightly astigmatic and their centroids are slightly shifted due to the differences in their tilt mentioned in the last paragraph. The off-diagonal elements have much lower amplitudes, about 0.0037 and have interesting form, being dark down the middle, and changing sign crossing the middle. All these arise at the fold mirror. These off-diagonal PSF images are referred to as the ghost PSFs.

Table 1. Parameters associated with the imaging calculation.

\begin{tabular}{|l|l|}
\hline Wavelength & $800 \mathrm{~nm}$ \\
\hline Image space F/\# & 8 \\
\hline Entrance pupil diameter & $2.4 \mathrm{~m}$ \\
\hline Effective focal length & $19.236 \mathrm{~m}$ \\
\hline Number of rays across entrance pupil & 65 \\
\hline Number of rays across the Jones pupil array after padding with zeros & 513 \\
\hline Spacing in the ARM and PSM viewing from object space & 9.0 milliarcsec \\
\hline
\end{tabular}


With unpolarized illumination, the incident $X$ and $Y$-polarizations are incoherent with respect to each other, which is the definition of unpolarized light. As a result, the output components $A R M_{X X}\left(X\right.$ in $X$ out) and $A R M_{Y X}(X$ in $Y$ out $)$ are coherent with each other but incoherent with $A R M_{X Y}$ and $A R M_{Y Y}$. So for unpolarized illumination, the two output $X$-components in the ARM are incoherent with respect to each other, as are the two output $Y$-components. So the PSF for an unpolarized source has four additive components $I=I_{X}+I_{Y}=\left(\left|A R M_{X X}\right|^{2}+\left|A R M_{X Y}\right|^{2}\right)+\left(\left|A R M_{Y X}\right|^{2}+\left|A R M_{Y Y}\right|^{2}\right)$. The polarization structure of the generic telescope PSF is explored further in the next section.

\section{MUELLER MATRIX POINT SPREAD MATRIX}

The Mueller matrix Point Spread Matrix (PSM), a $4 \times 4$ the Mueller matrix generalization of the PSF, defines the distribution of flux and polarization in the image of an incoherent point source, such as a star. ${ }^{[1]}$. This PSM is calculated by converting the ARM's Jones matrix functions into a Mueller matrix function. ${ }^{[24]-[26]}$ Figure 8 shows the telescope's PSM

The Stokes parameter image in the first column $(\mathrm{m} 00, \mathrm{~m} 10, \mathrm{~m} 20, \mathrm{~m} 30)$ inside the red rectangle is the PSF for unpolarized illumination. The PSF of an unpolarized star is not unpolarized since $\mathrm{m} 10, \mathrm{~m} 20$ and $\mathrm{m} 30$ are not zero. The $Q$ component's $\left(\mathrm{m} 10, \sim 4.7 \times 10^{-2}\right)$ predominantly comes from the fold mirror's diattenuation, which is reflecting more $0^{\circ}$ ( $s$-polarized) light than $90^{\circ}$ polarized light. The smaller $U$ component $\left(\mathrm{m} 20, \sim 4.36 \times 10^{-3}\right)$ is mostly generated from diattenuation contributions at $45^{\circ}$ and $135^{\circ}$ from the primary and secondary; this is seen in Figure 4's first two panels. The small ellipticity (the $V$ component, m30) occurs because weakly polarized light reflected from the primary and secondary interacts with the retardance from the fold mirror.

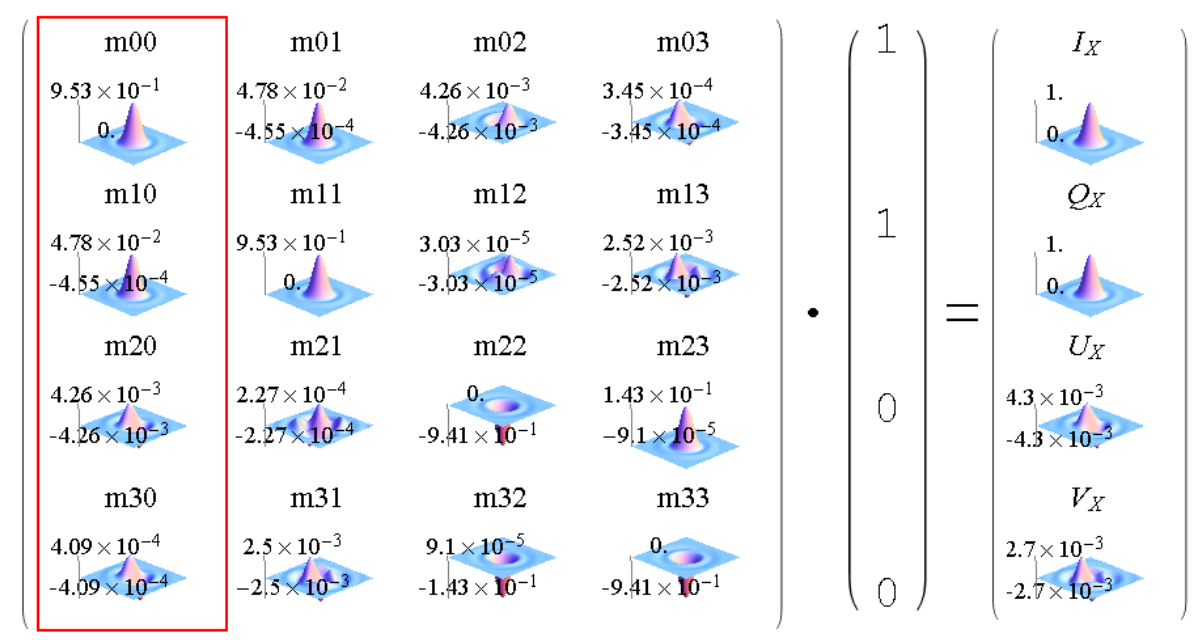

Figure 8. The point spread matrix (PSM) (left, in large brackets) here is shown operating on an $X$-polarized incident beam (Stokes parameters $(1,1,0,0)$ (middle brackets)) and results in a polarized PSF (right brackets). This resulting $4 \times 1$ Stokes image (right) has components $\left(I_{X}, Q_{X}, U_{X}, V_{X}\right)$. The subscript $X$ is used to show these Stokes parameters resulted from incident $X$-polarized light. Each matrix element's normalized magnitude is shown in a vertical scale. The $U_{X}$ image indicates a small variation of polarization orientation. $V_{X}$ shows the image also has small ellipticity variations. The red box ( $\mathrm{m} 00$, $\mathrm{m} 10, \mathrm{~m} 20, \mathrm{~m} 30)$ is the Stokes parameter image $(I, Q, U, V)$ for a collimated beam of unpolarized incident light, such as light incident from an unpolarized star.

This PSM distribution raises concerns for the application of telescopes like this to exoplanet detection, where a dark null in the exoplanet region is needed very close to a bright star. The outer portions of the PSF are strongly affected by the light coupled into orthogonal components because the $A_{X Y}$ and $A_{Y X}$ Jones pupil components are highly apodized; see Figure 6 (a). Comparing the PSM terms $I_{X X}$ and $I_{Y X}$; the first is nearly diffraction limited, the other is about twice as large. These PSF terms are calculated using the resultant Stokes image components as:

$$
I_{X X} \propto \frac{I_{X}+Q_{X}}{2} \quad \text { and } \quad I_{Y X} \propto \frac{I_{X}-Q_{X}}{2} .
$$

$I_{X X}$ and $I_{Y X}$, the two terms in Eq. (6), are compared in Figure 9. The peak of $I_{Y X}$ is about $10^{-5}$ of the peak of $I_{X X}$. In imaging applications needing contrast ratios of $10^{-8}$ or greater, this "ghost PSF" could be a significant detriment. 
To compare the diagonal and ghost components further, Figure 9 and Figure 10 shows the flux along an $x$-axis crosssection through the centers of the two PSFs in log scale; this is along the plane drawn through the two images shown in Figure 9. The ghost PSF's light spreads away from the center, which can also be seen in Figure 10. The Airy disk's zeros of $I_{X X}$ are not at the same location as the zeros for the cross-coupled term $I_{Y X}$. Thus the zeros, the dark rings, of $I_{X X}$ are washed out by the light leakage from $I_{Y X}$. Furthermore, the PSF of $I_{Y X}$ cannot be corrected by wavefront compensation for either the $X X$ or $Y Y$-components alone, because larger spread of the $I_{Y X}$ component is due to $I_{Y X}$ 's apodization, not its wavefront (Figure 6). $I_{Y X}$ can be removed with an $x$-oriented linear polarizer near the image; this can pass $I_{X X}$ and remove $I_{Y X}$, but the other ghost $I_{X Y}$, will now slip through; thus a simple polarizer will not completely correct this polarization aberration.

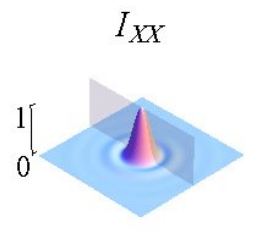

(a)

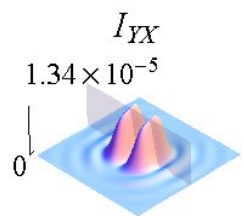

(b)

Figure 9. PSF of $I_{X X}$ and $I_{Y X}$ normalized to the peak of $I_{X X}$. Note the enlarged size of $I_{Y X}$.

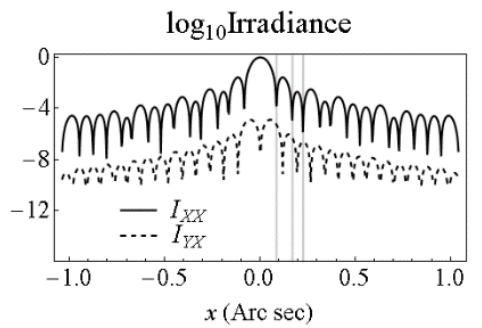

(a)

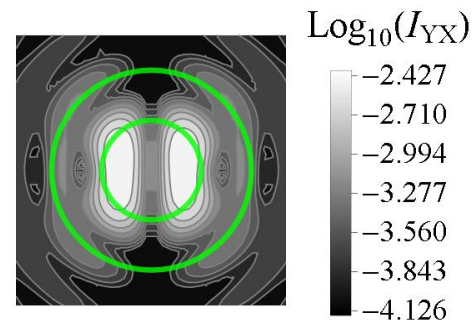

(b)

Figure 10. (a) $\log _{10} \mathrm{PSF} x$-cross-sections through the $I_{X X}$ and $I_{Y X}$ images. The dashed and solid curves show $I_{Y X}$ and $I_{X X}$ respectively. The nearest three dark rings in the Airy disk, the first three minima of $I_{X X}$, are close to local maxima of the $I_{Y X}$. On the average, the polarization coupled $I_{Y X}$ flux is about $10^{-4}$ below $I_{X X}$. (b) $I_{Y X}$ shown in $\log _{10}$ contour with its scale on the right ranging from -2.4 to -4.1 , normalized to the peak intensity of $I_{X X}$. The location of the first and second Airy dark rings of $I_{X X}$ are depicted with green circles.

The shape of $I_{Y X}$ indicates that the $I_{\mathrm{X}}$ and $Q_{\mathrm{X}}$ Airy disks (with small deviations) are not aligned, but shifted. The image plane irradiance distribution for the $I_{Y X}$ term sits beneath the Airy diffraction pattern of the $I_{X X}$ term. Figure 10 (a) shows a slice normal to the axis at the RMS best focus through the PSF for $I_{X X}$ and for $I_{Y X}$ in Figure 9. Figure 10 (b) shows a highdynamic range image of the irradiance across the focal plane in the vicinity of the core of the PSF for $I_{Y X}$. The first and second zeros of the Airy diffraction pattern of $I_{X X}$ are shown as concentric green circles superposed on Figure 10 (b). Note how these dark rings coincide with non-zero $I_{Y X}$.

The Stokes parameter PSF for the $X$-polarized component of an incident beam is shown in the right column in Figure 8, with flux $I_{X}=I_{X X}+I_{Y X}$. Similarly, the PSF of $I_{Y}$ (a $Y$-polarized incident beam) is found by matrix multiplying the PSM on the right by the Stokes parameters $(1,-1,0,0)$, yielding $I_{Y}=I_{Y Y}+I_{X Y}$. Finally the unpolarized incident light PSF is $\left(I_{X}+\right.$ $\left.I_{Y}\right) / 2$ which can also be calculated by matrix multiplying the PSM on the right by the unpolarized Stokes parameters $(1,0$, $0,0)$.

For unpolarized starlight or other nearly unpolarized sources, a "generic" optical system like Figure 2 creates a PSF which is best understood as the sum of two nearly Airy diffraction patterns (nearly because of some wavefront aberration and apodization effects), $I_{X X}$ and $I_{Y Y}$, plus two "ghost" PSFs, $I_{Y X}$ and $I_{Y X}$, which result from polarization cross-talk, the Jones pupil's off-diagonal elements.

\section{LOCATION OF THE PSF IMAGES}

Picture an imaging Stokes polarimeter measuring the example telescope's PSF of an unpolarized star. The PSFs for the $X$ polarized, $I_{\mathrm{X}}=I_{\mathrm{XX}}+I_{\mathrm{YX}}$, and $Y$-polarized light, $I_{Y}=I_{\mathrm{XY}}+I_{\mathrm{YY}}$, at the focal plane are close to the Airy diffraction pattern 
because the polarization-induced wavefront aberration, $\phi_{X X}$ and $\phi_{Y Y}$ in Figure 6, is less than 8 milli-waves, and the amplitude apodization is less than 0.015 . But these two PSF images peaks for $I_{\mathrm{X}}$ and $I_{Y}$ are shifted by 0.625 masec. Figure 11 contains PSF cross-section through the maxima plotted for $I_{X}, I_{Y}$, and $I_{X}-I_{Y}$ (the Stokes $Q$ image). As mentioned earlier, the shift between the $I_{\mathrm{X}}$ and $I_{Y}$ PSFs is due to the slope differences of the $p$ and $s$ - Fresnel phases, (blue and red tangent lines in Figure 3 (b)) which is the cause of the overall linear variations in $\phi_{X X}$ and $\phi_{Y Y}$. Their difference $Q=I_{X}-I_{Y}$ is sheared from $I_{X}$ and $I_{Y}$ by 5.8 masec, as shown in Figure 11, and is due to the shift between $I_{X}$ and $I_{Y}$. These are listed in Table 2. An ellipse was fitted to the PSF at the half power points to calculate an ellipticity of the PSF.

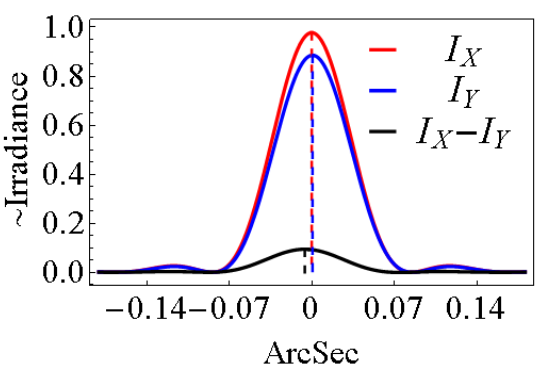

Figure 11. The cross-section profiles of the $I_{\mathrm{X}}$ and $I_{Y}$ PSF images, one for each polarization and the profile of their difference are shown in red and blue in arc seconds from the center of the PSF. The black line shows Stokes Q image, the difference between the two PSFs.

Table 2. The characteristics of the example telescope's PSF shape are described by the following parameters: the PSF's flux, the radius of encircled energy, the PSF shears and the PSF ellipticity for $X$ and $Y$-polarized incident light.

\begin{tabular}{|ll|}
\hline PSF shear in object space: & \\
\hline Between $I_{X}$ and $I_{Y}$ & 0.625 masec \\
Between $I_{X}$ and $\left(Q=I_{X}-I_{Y}\right)$ & 5.820 masec \\
\hline Flux in PSF: & \\
\hline flux of $I_{Y X}$ & $0.0048 \%$ \\
\hline flux of $I_{X X}$ & \\
flux of $I_{Y Y}$ & $90.6 \%$ \\
\hline flux of $I_{X X}$ & \\
flux of $I_{Y X}$ & $0.0046 \%$ \\
\hline flux of $I_{X X}$ & \\
Peak of $I_{Y}$ & \\
\hline Peak of $I_{X}$ & $90.6 \%$ \\
Peak of $\left(I_{X}-I_{Y}\right)$ & \\
\hline Peak of $I_{X}$ & Peak of $Q$ \\
\hline Radius of $90 \%$ encircled energy in object space: & Peak of $I_{X}$ \\
\hline r $_{X X}=$ r $_{Y Y}$ & \\
\hline r & r $X .6 \%$ \\
\hline Ellipticity of PSF: & \\
\hline $\begin{array}{l}\text { Unpolarized incident light } \\
X \text {-polarized incident light }\end{array}$ & \\
\hline -polarized incident light & 0.15 arc sec \\
\hline
\end{tabular}

In astronomical applications involving the precise measurement of the location of the centroid of the PSF, distortions of the shape of the PSF are important. Most systems incorporate multiple folds, for example in references [28] and [29]. These relay optics with multiple folds may increase the shear between PSF's polarization components. The variation of linear phase across the pupil, $\phi_{X X}$, and $\phi_{Y Y}$, seen in Figure 6, is approximately linear, thus the shear between polarization 
components is linear in the F/\#. As [29] showed, across the FOV, variations of PSF ellipticity and orientation are expected from polarization aberration. The Fresnel polarization aberrations, unless corrected, may affect our ability to characterize exoplanets using space telescopes.

\section{POLARIZATION ABERRATION MITIGATION}

The polarization aberrations of an optical system can be changed by a variety of methods. ${ }^{[31]}$ It is beyond the scope of this paper to explain such methods in detail. These polarization aberrations do tend to have small magnitude, often with constant, linear, quadratic, and other low order variations. ${ }^{[2],[4]}$ The following lists several mitigation approaches. ${ }^{[2],[4]}$ Because they are small, many of the following cures may be worse than the problem.
(A) Reducing angles of incidence,
(B) Reducing coating polarization,
(C) Compensating polarization elements,
(D) Crossing fold mirrors,
(E) Compensating optical elements.

\section{POLARIZATION ABERRATION EXPANSION AND SCALING RELATIONS}

A polarization aberration expansion, like a wavefront aberration expansion, is useful to understand how the system changes with variations to numerical aperture, angles of incidence, coating changes and the like. The polarization aberration of the example telescope's on-axis field is described accurately by a polarization aberration expansion containing constant, linear, and quadratic terms where

$$
\mathbf{J}=\sigma_{0}+\sigma_{1}\left[\frac{\left(d_{0}+i \Delta_{0}\right)-\left(d_{1}+i \Delta_{1}\right) \rho \sin \phi+\left(d_{2}+i \Delta_{2}\right) \rho^{2} \cos 2 \phi}{2}\right]+\sigma_{2} \frac{\left(d_{1}+i \Delta_{1}\right) \rho \cos \phi+\left(d_{2}+i \Delta_{2}\right) \rho^{2} \sin 2 \phi}{2}
$$

with aberration coefficients for the retardance, diattenuation, amplitude, and wavefront for the Jones pupil of the telescope end-to-end from Figure 7. This is similar to fitting the diattenuation and retardance with the three lowest order Zernike polynomials. These coefficients in the expansion will be determined by curve fitting to the Jones pupil polarization ray trace data yielding the values of Table 3 . The Pauli matrix definitions are

$$
\boldsymbol{\sigma}_{0}=\left(\begin{array}{ll}
1 & 0 \\
0 & 1
\end{array}\right), \quad \boldsymbol{\sigma}_{1}=\left(\begin{array}{cc}
1 & 0 \\
0 & -1
\end{array}\right), \quad \boldsymbol{\sigma}_{2}=\left(\begin{array}{ll}
0 & 1 \\
1 & 0
\end{array}\right), \quad \boldsymbol{\sigma}_{3}=\left(\begin{array}{cc}
0 & -i \\
i & 0
\end{array}\right)
$$

Table 3. Polarization aberration coefficients for telescope's Jones pupil in figure 6 .

\begin{tabular}{ll}
\hline & Polarization aberration coefficients \\
\hline Diattenuation & $d_{0}=0.050, d_{1}=-0.008, d_{2}=-0.007$ \\
Retardance & $\Delta_{0}=-0.151, \Delta_{1}=-0.023, \Delta_{2}=-0.022$ \\
Amplitude & $a_{0}=0.806, a_{1}=-0.002, a_{2}=0.0000$ \\
Wavefront & $w_{0}=2.492, w_{1}=-0.004, w_{2}=0.000$ \\
\hline
\end{tabular}

Note all of the polarization aberration coefficients are much less than one, so they qualify as small. Scalar terms for the wavefront aberration $W(\rho, \phi)$ and apodization $A(\rho, \phi)$ are combined with $\mathbf{J}$ for a full second order Jones pupil description,

$$
A(\rho, \phi) e^{i 2 \pi W(\rho, \phi) / \lambda} \mathbf{J} .
$$

The necessary wavefront aberration terms go to second order $W(\rho, \phi)$,

$$
W(\rho, \phi)=w_{0}+w_{1} \rho \sin \phi+w_{2} \rho^{2},
$$


including piston $w_{0}$, tilt $w_{1}$, and, defocus $w_{2}$. These result from the phase variations of the average Fresnel coefficients with angle. The averaged Fresnel equations also generate small polarization independent amplitude variations expressed with similar coefficients $a_{0}, a_{1}$, and $a_{2}$ as

$$
A(\rho, \phi)=a_{0}+a_{1} \rho \sin \phi+a_{2} \rho^{2} .
$$

With these functions and twelve aberration terms, the polarization aberration approximation to the Jones pupil fits within $1 \%$.

With a closed form polarization aberration expression, the behavior of the telescope can be parameterized. For example, the diattenuation at the center of the pupil corresponds to the diattenuation piston term with magnitude $d_{0}$. This arises only from the fold mirror; the primary and secondary mirrors do not contribute diattenuation at the center of the pupil. The coefficient $d_{1}$ is close to the value of the average diattenuation, averaging over the pupil. From the quadratic variation of the coating diattenuation about normal incidence, it is seen that the average diattenuation (diattenuation piston value) characterized $d_{0}$ by is quadratic in the fold mirror's fold angle. More on the use of this polarization aberration expansion to generate scaling relationships in found in Breckinridge [32].

\section{REFERENCES}

[1] Kuboda, H. and Inoué, S., "Diffraction images in the polarizing microscope," J. Opt. Soc. Am 49, 191-198 (1959).

[2] Chipman, R. A., "Polarization analysis of optical systems I," Opt. Eng. 28, 90-99 (1989).

[3] Bruegge, T. J., "Analysis of Polarization in Optical Systems," Proc. SPIE 1166, 165-176 (1989).

[4] Chipman, R. A., "Polarization analysis of optical Systems II," Proc. SPIE 1166, 79-99 (1989).

[5] Waluschka, Eugene, "Polarization Ray Tracing," Proc. SPIE 0891, 104 (1988).

[6] Wolff, L. B. and Kurlander, D. J., "Ray Tracing with Polarization Parameters," IEEE Computer Graphics and Appl., 44-55 (1990).

[7] Yun, G., Crabtree, K., and Chipman, R., "Three-dimensional polarization ray-tracing calculus I: definition and diattenuation," Appl. Opt. 50, 2855-2865 (2011).

[8] Yun, G., McClain, S., and Chipman, R., "Three-dimensional polarization ray-tracing calculus II: retardance," Appl. Opt. 50, 2866-2874 (2011).

[9] Urbanczyk, W., "Optical Imaging Systems Changing the State of Light Polarization,” Optik B, 301-309 (1984).

[10] Urbanczyk, W., "Optical Transfer Functions for Imaging Systems Which Change the State of Light Polarization," Opt. Acta. 33, 53-62 (1986).

[11] McGuire, J. P. and Chipman, R. A. "Diffraction image formation in optical systems with polarization aberrations 1. Formulation and example," JOSA A 7, 1614-1626 (1990).

[12] McGuire, J. P. and Chipman, R. A., "Diffraction image formation in optical systems with polarization aberrations. II: Amplitude response matrices for rotationally symmetric systems,” J. Opt. Soc. Am. A 8(6), (1991).

[13] Mansuriper, M., "Effects of High-numerical-aperture Foucsing on the State of Polarization in Optical and Magnetooptical Data Storage Systems.” Appl. Opt. 30(22), 3154-3162 (1991).

[14]Dorn, R., Quabis, S., and Leuchs, G., "Sharper focus for a radially polarized light beam," Phys. Rev. Lett. 91, 233901 (2003).

[15] Tu, Y., Wang, X., Li, S., and Cao, Y., “Analytical approach to the impact of polarization aberration on lithographic imaging," Opt. Lett. 37, 2061-2063 (2012).

[16] Chipman, R. A., Polarization Aberrations, PhD dissertation, College of Optical Sciences, University of Arizona, Tucson AZ (1987).

[17] McGuire Jr., J. P. and Chipman, R. A., "Polarization Aberrations in Optical Systems" Proc. SPIE 818, 240-245 (1987).

[18] McGuire, J. P. and Chipman, R. A., "Polarization Aberrations 1. Rotationally symmetric optical systems," Applied Optics 33(22), 5080-5100 (1994).

[19] McGuire, J. P. and Chipman, R. A., "Polarization aberrations. 2, Tilted and decentered optical systems," Applied Optics 33(22), 5101-5107 (1994).

[20] Hansen, E. W., “Overcoming Polarization Aberrations in Microscopy,” Proc. SPIE 891, 190-197 (1988).

[21] Chipman, R. A. and Chipman, L. J., "Polarization aberration diagrams," Opt. Eng. 28(2), 100-106 (1989).

[22] Shriebak, M., Inoue, S., and Oldenbourg, R., "Polarization aberrations caused by differential transmission and phase shift in high-numerical-aperture lenses: theory, measurement and rectification," Opt. Eng. Bellingham 41, 943 (2002). 
[23] Beckley, A. and Brown, T., "Imprinting Aberrations in the Stokes Parameters of a Focal Spot," in International Optical Design Conference and Optical Fabrication and Testing, paper IWA5, OSA Technical Digest (CD) (Optical Society of America, 2010).

[24] Goldstein, D., Polarized Light, 3d Edition, CRC Press (2010).

[25] Gil, J. J., "Polarimetric characterization of light and media," The European Physical Journal Applied Physics 40, 147 (2007).

[26] Chipman, R. A., Chapter 14 Mueller Matrices in Handbook of Optics (Vol I), Michael Bass editor, McGraw-Hill (2009).

[27] Ruoff, J., and Totzeck, M., "Orientation Zernike polynomials: a useful way to describe the polarization effects of optical imaging systems," J. Micro/Nanolith. MEMS MOEMS., 8(3), (2009).

[28] Goullioud, R., Zhao, F., Tang, H., and Wu, J., "The WFIRST-AFTA coronagraph design update," SPIE 9143, 91430 S (2014).

[29] Witzel, G., Eckart, A., Bucholtz, R. M., et al., "The Instrumental Polarization of the Nasmyth focus polarimatric differential imager NAOS/CONICA (NACO) at the VLT," A\&A 525 A130, 1-15 (2011).

[30] Clark, Natalie, and Breckinridge, James B., "Polarization compensation of Fresnel aberrations in telescopes," Proc. SPIE 8146, 814600 (2011).

[31] Maymon, P. W. \& Chipman, R. A., "Linear polarization sensitivity specifications for spaceborne instruments," Proc. SPIE, 1746, 148-156 (1992).

[32] Breckinridge, J. B., Lam, W. S., and Chipman, Russell A. "Polarization Aberrations in Astronomical Telescopes: The Point Spread Function", Publications of the Astronomical Society of the Pacific 127, 445-468 (2015). 